Original Article

\title{
HOMOLOGY MODELING OF SUBCUTANEOUS FILARIASIS DHFR PROTEINS
}

\author{
KAVITA CHANDRAMORE \\ Department of Pharmaceutical Science and Technology, Institute of Chemical Technology, Matunga, Mumbai \\ Email: prateek.jain246@gmail.com
}

Received: 20 Aug 2017, Revised and Accepted: 13 Oct 2017

\section{ABSTRACT}

Objective: A systematic technique for protein modeling offers great assistance in the study of protein function, dynamics, interactions with ligands, and other proteins and even in drug discovery and drug design. Subcutaneous filariasis is rare parasitic disease caused by Loa Loa (eye worm) and monosonallastreptoscerca species.

Methods: The present study develop three dimensional structure of dihydrofolatereductase present in Loa loa species. For this purpose knowledge based homology modeling is used by using Schrodinger Glide 5.6 software.

Results: The procedure involves alignment that maps residues in the query sequence to residues in the template sequence to generate structural model of target, which was further refined and final result validated by using Ramchandran plot.

Conclusion: In ramchandran plot majority of the amino acids are in the phi-psi distribution and the develop model is reliable and of good quality.

Keywords: Subcutaneous filariasis, Loa Loa, Homology modeling

(C) 2017 The Authors. Published by Innovare Academic Sciences Pvt Ltd. This is an open access article under the CC BY license (http://creativecommons.org/licenses/by/4.0/)

DOI: http://dx.doi.org/10.22159/ijcpr.2017v9i6.23433

\section{INTRODUCTION}

Dihydro-folate-reductase (DHFR) is a key enzyme in folate metabolism; it plays a major role in the biosynthesis of nucleic acid throught the catalysis of the NADPH reduction of 7, 8-dihydrofolate to $5,6,7$, 8-tetrahydrofolate. DHFR protein is main target in subcutaneous filariasis. This inhibitor also have anticancer, antibacterial and even antimalarial activity, which is collectively known as anti-folates. Loa loa is a thread like warm that lives under the skin in subcutaneous fat causing loiasis [1]. It is one of three parasitic filarial nematodes that cause loiasis in human. The other two are monsonellastreptocerca and onchocercavolvulus [2]. Homology modeling also known as comparative modeling of protein is the technique which allows to construct an unknown atomicresolution model of target protein from its amino acid sequence and an 3D structure of a related homologous protein (the template) [3]. Therefore the present study focused on to build a high quality of protein 3D structure for subcutaneous filariasis DHFR protein.

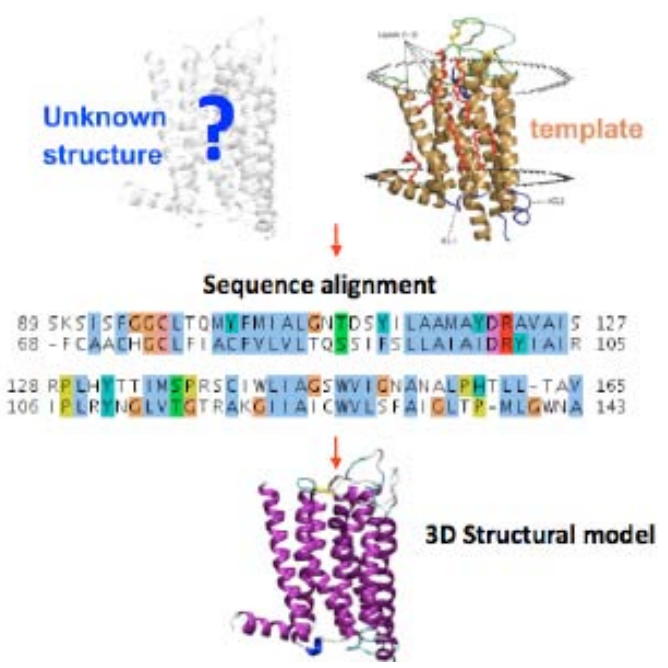

Fig. 1: Homology modeling

\section{Homology modeling}

The protocol to the model building by homology modeling (scheme 1) involves template identification, alignment of the sequences, generation of the model coordinates, optimization, and validation of the model [4]. The process start with getting the amino acid sequence then identify related homologous sequence as template. In next step download the template sequence, the sequence alignment between target and selected template, then build up the model and optimize and evaluate (fig. 1) [5-6].

Getting the sequence of amino acid

Identify the relative homologous sequence as a templet

Download the templet sequence

Sequence aligment between target and templet sequence

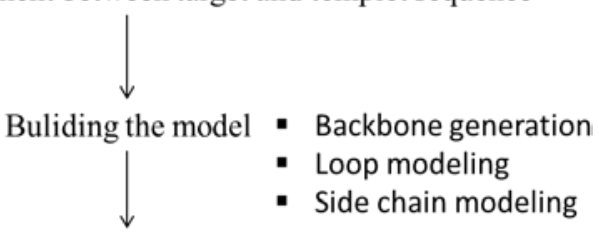

Model optimization and evaluation

Scheme 1: Protocol for homology modeling

\section{MATERIALS AND METHODS}

1) Amino acid sequence and identify related homologous sequence: Amino acid sequence of DHFR protein for subcutaneous filariasis was downloaded from www.unipro.org. And DHFR malaria select as 
template which is homologous to target protein. Template sequence also download from same website.

2) Sequence alignment: A search with BLAST against this database will give a list of known protein structures that match the sequence. BLAST will provide a list of possible templates for the unknown structure. In sequence alignmentalign the target sequence and template sequence.

3) Building the model: In this step factor involve to build the model is: 1) backbone generation 2) loop modeling 3) side chain modeling. There are two type of model: knowledge base model and energy based model. By using knowledge based model subcutaneousfilariais protein was build up. Further efficiency was improve by using refining loops. There are three refinement task available in refine structure stepi. e refine loops, predict side chain and minimization.

4) Evaluation: The model was further evaluated by using ramachandran plot.

\section{RESULTS AND DISCUSSION}

1) Basic local alignment search tool: One of the most widely used algorithms for comparing primary biological sequences, such as aminoacid sequences, is BLAST (Basic Local Alignment Search Tool). In this search the DHFRloaloa amino acid sequence compare with DHFR malaria sequence shown in fig. 2.

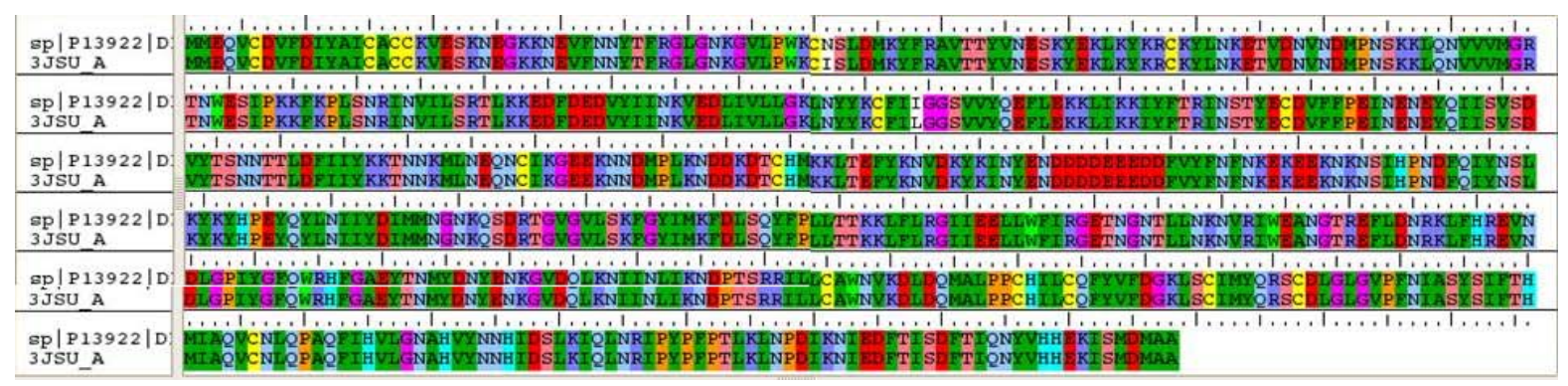

Fig. 2: Basic local alignment search tool

2) Template selection: After BLAST, result as a list of 3D protein structures ordered according to their "sequence identity percentage" with the target sequence. The quality of the homology model is dependent on the quality of the sequence alignment and template structure. Model quality declines with decreasing sequence identity. Highest percentage identity template was selected shown in fig. 3.

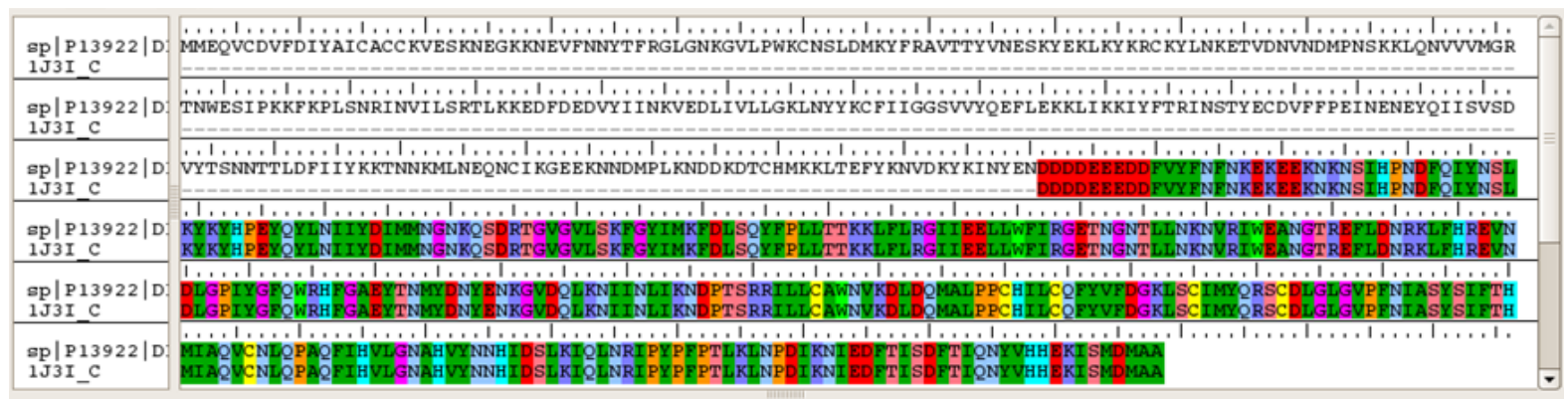

Fig. 3: Template selection

3) Sequence Alignment: For model development, sequence alignment file between DHFR loaloaand the selected DHFR malaria template sequence was carried out shown in fig. 4.

4) Building model: The Build Structure program builds insertions, closes gaps, and predicts side-chain conformations.
The structure produced in the Build Structure step is likely to represent only a local energy minimum and not the global minimum.

Therefore, regions with gaps in the alignment are likely to require refinement in the Refine Structure step shown in fig. 5.

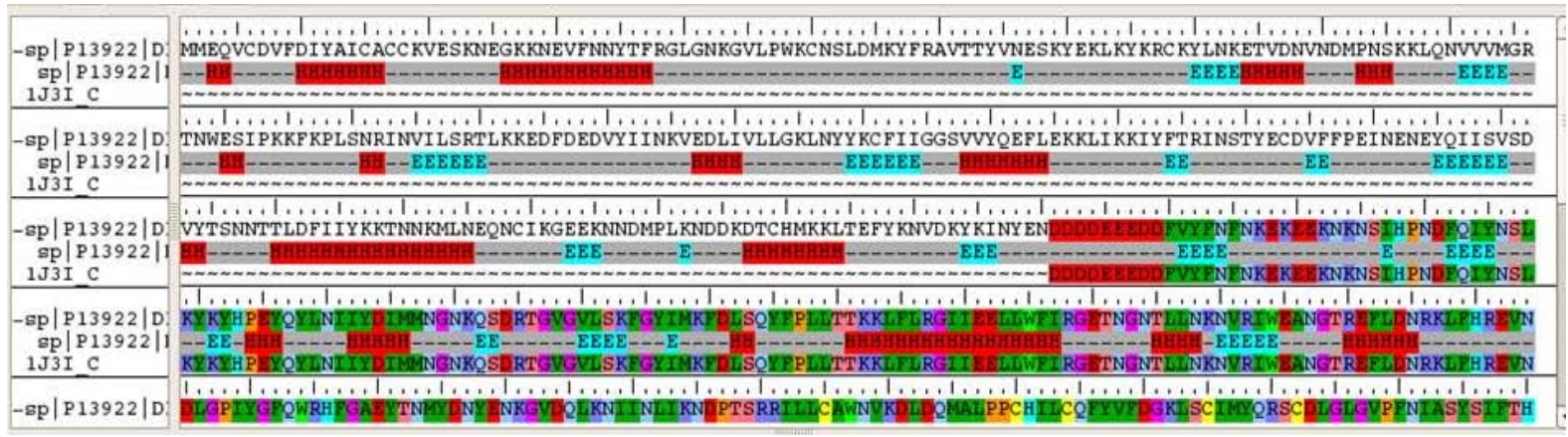

Fig. 4: Sequence alignment 


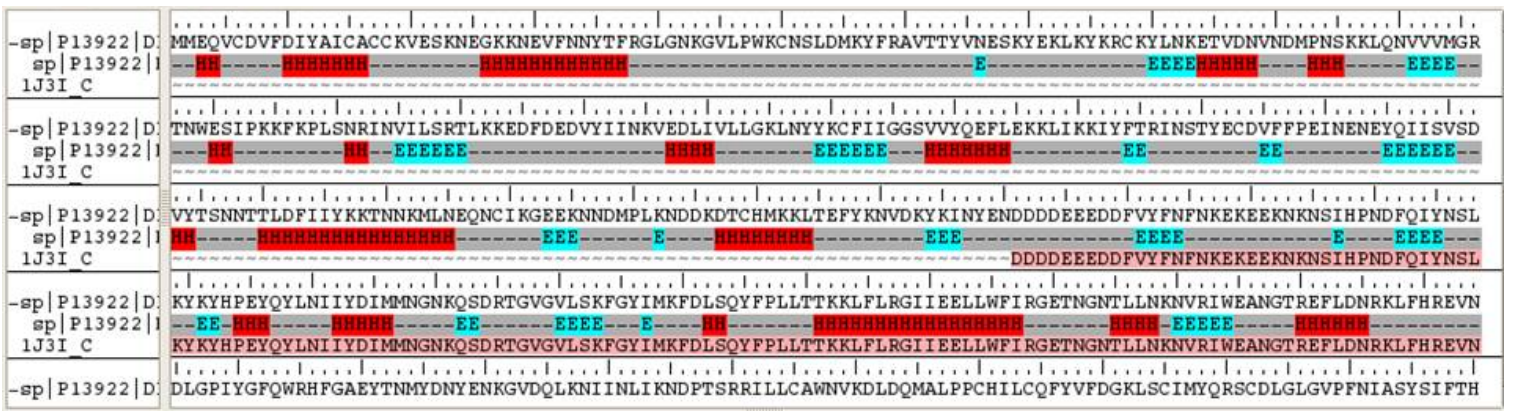

Fig. 5: Building model

5) Refine model: To improve the structure most efficiently the refinement of model required. There are three refinement tasks was carried out in the Refine Structure step: Refine loops, Predict side chains, and minimize.

6) Model analysis: Minimize model was further evaluated by using ramachandran plot. The Ramachandran plot for the generated model and the template protein (DHFR malaria) is shown in fig. 6.2b. From the plot, it is revealed that majority of the amino acids are in the phi-psi distribution and the model is reliable and of good quality.

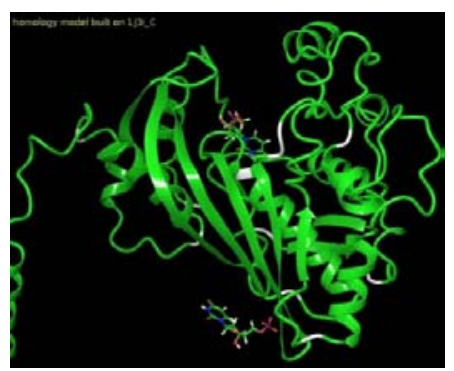

Fig. 6.1: a) Homology modelled structure of DHFR subcutaneous filariasis

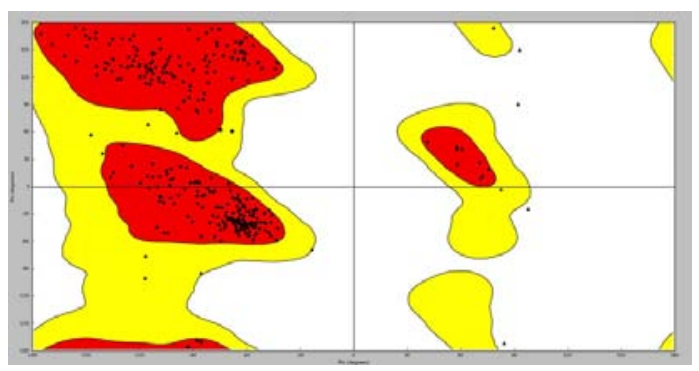

Fig. 6.2: b) Ramachandran plot of modelled structure of subcutaneous filariasis

\section{CONCLUSION}

The present study was carried out to examine the probable role of DHFR protein in subcutaneous filariasis. No X-ray crystal structure of DHFR subcutaneous filariasis was available at PDB. Hence, homology modelling approach was used to obtain the 3D-structure of DHFR subcutaneous filariasis protein using the structure of DHFR malaria as a template. The modelled structure was subjected to energy minimization and refinement. Refine structure was validated by using ramachandran plot. In ramchandran plot majority of the amino acids are in the phi-psi distribution and the develop model is reliable and of good quality.

\section{CONFLICT OF INTERESTS}

Declared none

\section{REFERENCES}

1. Spinello A, Luca Schifanella. Imported loa loafilariasis: three cases and a review of cases reported in non-endemic countries in the past $25 \mathrm{y}$. Int J Infect Dis 2012;16:e649e662.

2. ArchanaJayaraman, Kaiser Jamil. Homology modeling and docking studies of human alpha2-Adrenergic receptor subtype. J Comput Sci Syst Biol 2013;6:136-49.

3. Sakshi P, Vandana Saini. Homology modeling and molecular docking studies of human placental cadherine protein for it's role teratogenic effects of anti-epileptic drugs. Comput Biol Chem 2016;60:1-8.

4. Ankita S, Ravi Kumar Gundampati. Homology modeling and molecular docking of heme peroxidase form euphorbia tirucalli substrate specificity and thiol inhibitor interactions. J Mol Liquid 2016;220:383-94.

5. Rmeshkumar D, Sanjuktha M. Molecular modeling and docking studies on shrimp vitellogenin receptor and liganed mediated delivery system. Int J Res Drug Delivery 2012;2:11-4.

6. Paola Fossa, Elena Cichero. In silicoe valution of human small heat shock protein HSP27: Homology modeling, mutation analysis and docking studies. Bioorg Med Chem 2015; 23:3215-20. 